\title{
Can the Decreased Expression of Human Leukocyte Antigen Class I and II by Spermatozoa Lead to Recurrent Spontaneous Abortion?
}

\author{
Nasrin Sereshki ${ }^{1}$, Alireza Andalib ${ }^{1}$, Ataollah Ghahiri ${ }^{2}$, Ferdos Mehrabian ${ }^{2}$, Roya Sherkat ${ }^{2}$, Abbas Rezaei*1 \\ 1. Department of Immunology, School of Medicine, Isfahan University of Medical Sciences, Isfahan, Iran \\ 2. Department of Obstetrics and Gynecology, Al-Zahra Hospital, Isfahan University of Medical Sciences, Isfahan, Iran
}

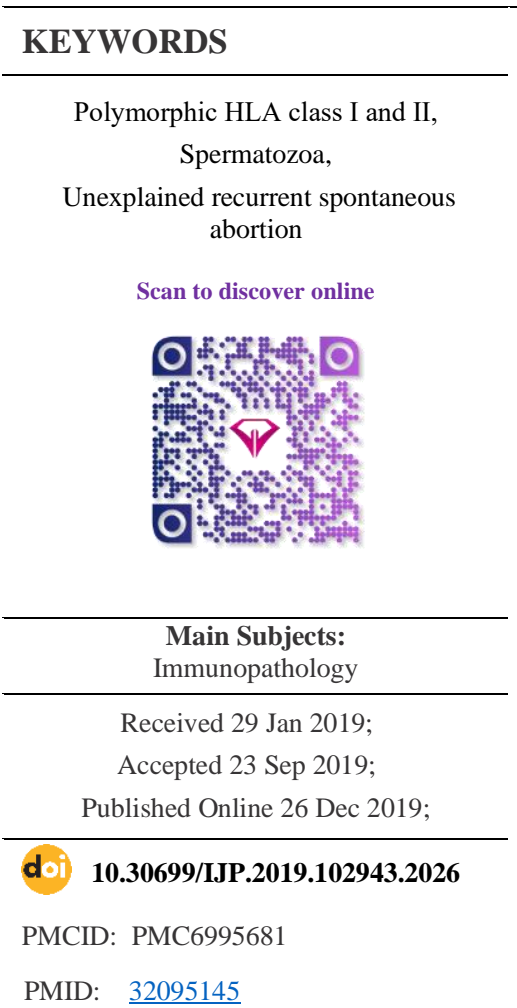

ABSTRACT

Background \& Objective: Unexplained recurrent spontaneous abortion (URSA) is defined as an unknown cause of occurrence of three or more clinically detectable pregnancy losses before 20 weeks of gestation, but it occurs presumably as a result of the immune system dysfunctions. We supposed that the disruption of semen or spermatozoa might be responsible for the dysfunction of the immune system in women with URSA. Semen and spermatozoa (as antigens) induce female reproductive tract (FRT) immunity. This stimulated immunity is necessary for pregnancy occurrence. The disruption of semen or spermatozoa can be a result of altering a variety of surface molecules on spermatozoa, especially polymorphic human leukocyte antigen (HLA) molecules or antigens. Despite the importance of HLA antigens in reproduction, to the best of our knowledge, no one has studied the relation of HLA expression between spermatozoa and URSA. Therefore, this paper aims to assess this relation.

Methods: Semen samples were collected from 15 URSA couples and 20 normal couples. After purification of normal spermatozoa, the HLA class I and II expressions were evaluated by flow cytometry methods.

Results: Results showed that the expression of both HLA class I and II by spermatozoa, in URSA couples, was significantly less than the control couples.

Conclusion: The decreased expression of polymorphic HLA class I and II by spermatozoa can be related to URSA occurrence.

Corresponding Information:

Abbas Rezaei, Assistant Professor, Department of Immunology, School of Medicine, Isfahan University of Medical Sciences, Isfahan, Iran Email: rezaei@mui.ac.ir

Copyright ( 9 2020. This is an open-access article distributed under the terms of the Creative Commons Attribution- 4.0 International License which permits Share, copy and redistribution of the material in any medium or format or adapt, remix, transform, and build upon the material for any purpose, even commercially.

\section{Introduction}

Recurrent spontaneous abortion (RSA) is a common problem among couples and is defined as the occurrence of three or more clinically detectable pregnancy losses that usually occur before 20 weeks of gestation (1). Some causes of RSA are uterine anatomical defects and infections, chromosomal aberrations, hormonal disorders, and immunological abnormalities (1). In about $50 \%$ of RSA couples, the causes of abortion still remain unknown, which is so-called unexplained recurrent spontaneous abortion (URSA). It has been understood that URSA occurs as a result of immune system dysfunctions (1). To the best of our knowledge, every investigation, in different aspects of immunity in URSA patients, has demonstrated immune system irregularity in these patients (2-6). We suppose that a reason for this immune irregularity and dysfunction might be the alteration of antigens that stimulate female reproductive tract (FRT) immune response. Antigen type is considered to be one of the most important factors for the stimulation of appropriate immunity. As antigens, semen and spermatozoa stimulate FRT immune system. After entering the vagina, semen and spermatozoa induce innate and adaptive immune responses in the FRT. The result of this activated immunity is memory regulatory lymphocytes and memory effector lymphocytes formation, which is directed to paternal antigens (7-10). Therefore, any alteration in seminal and spermatozoa antigenicity can result in disturbed immune response and consequently pregnancy aberration (7-10). As an allogeneic cell, spermatozoon can induce strong immune responses. Of the antigens, which make the allogeneic cells as a strong inducer of the immune response, are polymorphic human leukocyte antigen (HLA) antigens. However, there is conflicting data about polymorphic HLA class I and II expression by spermatozoa (11-17). We recently showed that ejaculated spermatozoa express both HLA class I and II. Moreover, some studies demonstrated that HLA molecules have some critical roles in pregnancy and its complications (18-21). 
Therefore, by considering the critical role of HLA antigens in reproduction and their main roles in the induction of immune response, we suppose that one of the most likely factors, which changes immunity in FRT after deposition of spermatozoa, is an abnormality in HLA antigen expression on spermatozoa. In other words, perhaps one of the causes, which disturbs immunity in URSA, is a change in HLA antigens. This change leads to unfavorable immune stimulation in FRT, and accordingly RSA occurs because there is no prepared condition for occurring and maintaining pregnancy. Given those aforementioned explanations, this paper aims to determine the HLA antigen expression level on spermatozoa in men whose wife suffer from URSA.

\section{Materials and Methods Subjects}

A total of 15 URSA couples (the range of age is from 24 to 40 years for men and 21 to 38 years for women) with at least three consecutive first-trimester abortions and 20 normal couples (the range of age is from 26 to 42 years for men and 27 to 40 years for women) with at least one child were included in this case-control study. The husband of each woman had normal semen status according to the criteria from the World Health Organization (WHO). All male partners did not have any history of genital tract disorder, such as a history of infection, undescended testis, inguinoscrotal surgery, genital trauma, or testicular torsion. Informed consent was obtained from all subjects. Semen samples were collected by masturbation after 2-3 days of sexual abstinence. After liquefaction at room temperature for $60 \mathrm{~min}$, sperm quality was assessed according to WHO standard guidelines (WHO, 1992). A sample with normal quality was selected to assess HLA expression. The protocol for this study was approved by the Ethics Committee of Isfahan University of Medical Sciences (Isfahan, Iran).

\section{Sperm Purification}

The AllGrad (LifeGlobal® Group, Canada) gradient technique was used for the purification of spermatozoa. Then, $2 \mathrm{~mL}$ of AllGrad Wash (LifeGlobal® Group, Canada) was added to the liquefied semen sample and centrifuged at $350 \mathrm{~g}$ for $10 \mathrm{~min}$. Next, the pellet was resuspended in $1 \mathrm{~mL}$ of AllGrad Wash. The gradient was prepared by pipetting $1 \mathrm{~mL}$ of AllGrad 90\% gradient into the bottom of the centrifuge tube and then layered by $1 \mathrm{~mL}$ of AllGrad $45 \%$ gradient carefully on top of the lower layer, followed by $1 \mathrm{~mL}$ of the spermatozoa suspension. The tubes were centrifuged at $400 \mathrm{~g}$ for 18 min. The spermatozoa pellet at the bottom of the centrifuge tubes was washed and re-suspended in AllGrad Wash.

\section{Flow Cytometry}

To assess HLA class I and II expression on the surface of spermatozoa, we used the flow cytometry method. Two tubes were prepared for each sample containing $1 \times 10^{6}$ spermatozoa. One was incubated with phycoerythrin (PE) mouse anti-human HLA-ABC (BD Pharmingen, USA), and the other one was incubated with PE mouse anti-human HLA-DR at room temperature for $30 \mathrm{~min}$. After two washes with AllGrad Wash (at $400 \mathrm{~g}$ for $5 \mathrm{~min}$ ), tubes were run through flow cytometer (BD FACSCalibur, USA). Data from at least 100,000 events were collected by using forward scatter (a logarithmic amplifier) and side angle of light scatter (a logarithmic amplifier). Fluorescence data were obtained via the logarithmic amplifier. FlowJo VX software was used to analyze the data. .
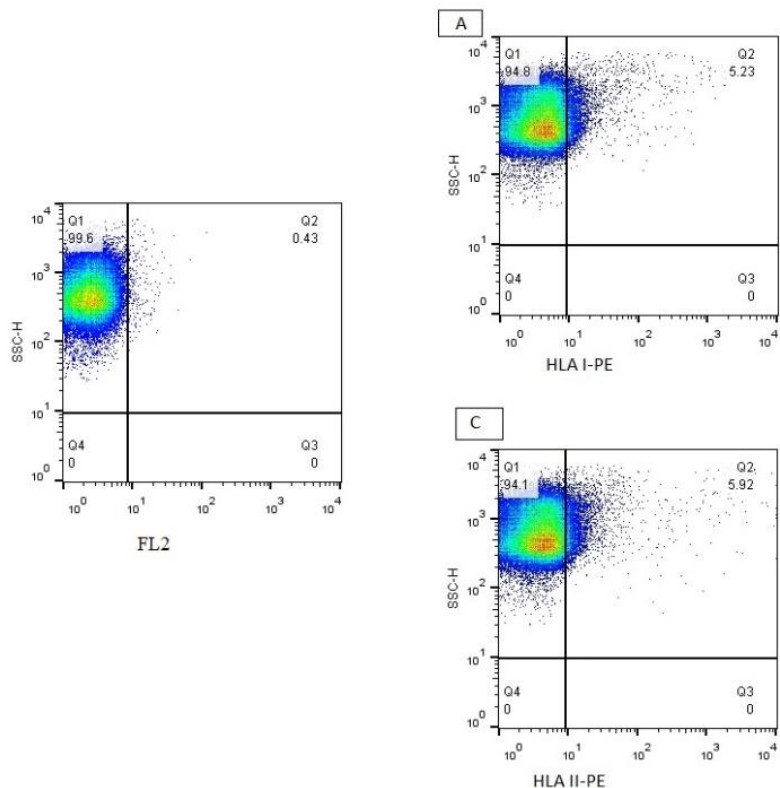
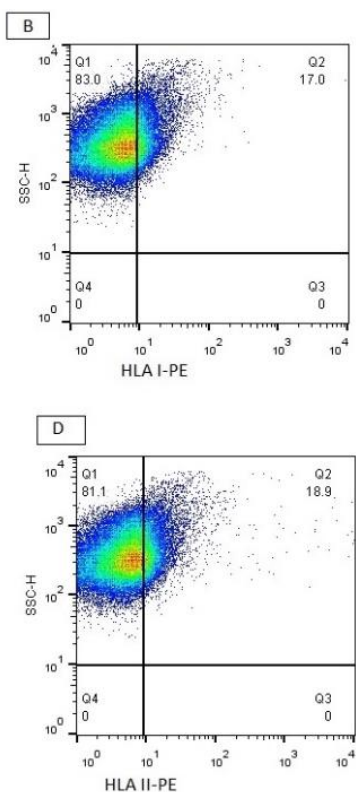

Fig. 1. Representative flow cytometry dot plots

A and C: flow cytometry dot plots in the RSA group; B and D: flow cytometry dot plots in the control group. 


\section{Statistical Analysis}

The Student's t-test was used to compare the differences between controls and URSA groups. Values were presented as mean \pm standard deviation (SD). A Pvalue of $<0.05$ was considered significant. All data analysis was performed by using SPSS 25 (SPSS Inc., Chicago, Illinois, USA).

\section{Results}

A flow cytometric assay was used to evaluate the HLA expression by spermatozoa (Figure 1). The results, which are presented in Figure 2, showed a significantly decreased mean percentage of the HLA class I and II expression by spermatozoa in the URSA group compared to the control group.

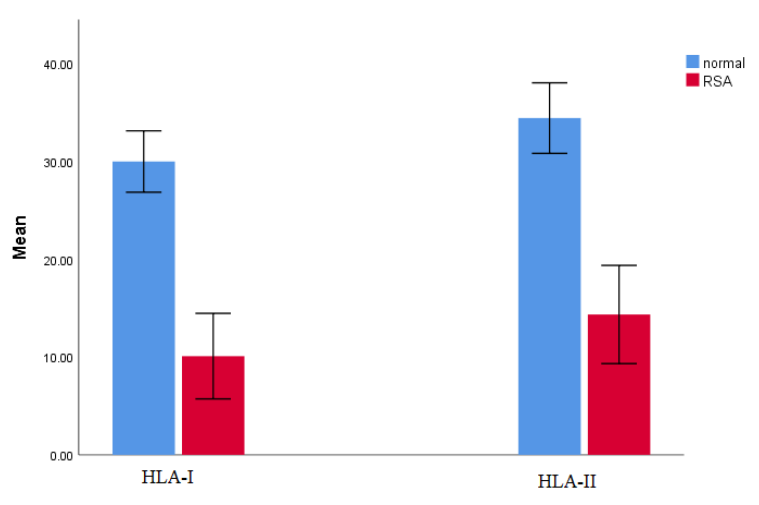

Fig. 2. The independent sample t-test was used to compare the mean percentage of HLA class I and II in the URSA and control groups. *: Considered significant in comparison with the control group. The mean percentage \pm SD of HLA class I in the URSA group is $10.1 \pm 2.2$, and in the control group is $29.9 \pm 1.6$; $\mathrm{P}$-value is 0.0001 . The mean percentage of HLA class II in the URSA group is $14.3 \pm 2.5$, and in the control group is $34.4 \pm 1.8$; P-value is 0.0001 .

\section{Discussion}

The alteration of spermatozoa antigenicity can lead to pregnancy complications, such as URSA (18). Accordingly, this study showed that there is a decreased expression of HLA class I and II on spermatozoa in RSA couples. To the best of our knowledge, no researcher has addressed the HLA class I and II expression on spermatozoa in couples with RSA.

There are old and very conflicting data in the field of polymorphic HLA expression by spermatozoa, and it has not yet been established whether polymorphic HLA expresses by spermatozoa. We think that using an insensitive and inappropriate technique for the detection of molecules with low expression can be the reason of this contradictory result. Among studies in this field, only a few of them evaluated the association between polymorphic HLA expression by spermatozoa and infertility $(16,17)$, and as far as we know, only one of them could show a correlation between HLA class II on spermatozoa and infertility (17). However, in the mentioned study, all samples (in both fertile and infertile groups) did not express HLA class II. The number of men whose spermatozoa expressed HLA class II was higher in the infertile group than in the fertile group. Therefore, it suggests that there is a positive correlation between HLA class II and infertility.

As we stated above, no study has been shown the relation between polymorphic HLA antigen and URSA. The present study is the first investigation that aimed to determine this relationship. This finding can be considered as a newly discovered cause of URSA. However, further investigations are necessary for determining the cause of alteration of HLA expression on spermatozoa and also determining why and how this alteration can eventuate RSA. The results of these investigations will support the discovery of a new therapy for URSA couples.

Regarding the induction of immunity by HLA antigens, most studies, especially in the field of transplantation, have shown that allogeneic HLA antigens induce the effector mechanism rather than the regulatory mechanism of the immune response. Also, this condition occurs in URSA women. Therefore, we hypothesized probably one of the reasons of enhanced effector immune responses in URSA couples may be a result of the increased expression of HLA antigen on spermatozoa. However, our study was opposite to our hypothesis. The evidence suggests that semen and spermatozoa must be able to stimulate immunity in the FRT strongly. Therefore, the expression of HLA antigens by spermatozoa, as a strong stimulator of immunity, may be essential for strong stimulation of immunity in the FRT. The fate of this immune response is a regulatory dominant immune response. We think that the decreased expression of HLA by spermatozoa can lead to disturbing this process and unsuccessful pregnancy.

In addition to the immune-stimulatory role of the HLA expression by spermatozoa, there is a possibility that HLA on spermatozoa has some roles in the fertility processes, such as sperm capacitation, acrosome reaction, and fusion with the oocyte. Nonetheless, further studies should be performed for determining the role of HLA antigens, their variants (presented by spermatozoa in the reproduction and pregnancy complication), and mechanism of action.

The limitation of our research is that a small sample size reduces the power of the study. Despite this limitation, we believe that our work could be a starting point for future investigations and introduction of new causes for URSA.

\section{Conclusion}

Our work has led us to conclude that the decreased expression of polymorphic HLA class I and II by spermatozoa can be related to occurring URSA. Although this study revealed only a relation between URSA and HLA expression by spermatozoa, further studies are necessary for determining the role and action mechanism of HLA antigens on spermatozoa in fertilization processes. However, we believe that our 
study is the first step toward discovering new causes for URSA and new therapy for this pregnancy complication.

\section{Acknowledgements}

The authors would like to thanks Dr. Hossein Motedayen and Mohadeseh Thogyani and Mohammad Sadegh Hesamian for their valuable assistance in guiding subjects for giving samples. This work was financially supported by Isfahan University of Medical Sciences. The number of the ethics committee approval letter is 395480 .

\section{Conflict of Interest}

The authors declared that there is no conflict of interest regarding the publication of this article.

\section{References}

1. Sereshki N, Gharagozloo M, Ostadi V, Ghahiri A, Roghaei MA, Mehrabian F, et al. Variations in T-helper 17 and regulatory $\mathrm{T}$ cells during the menstrual cycle in peripheral blood of women with recurrent spontaneous abortion. International Journal of Fertility and Sterility. 2014;8(1):5966.

2. Sasaki Y, Sakai M, Miyazaki S, Higuma S, Shiozaki A, Saito S. Decidual and peripheral blood CD4+CD25+ regulatory $\mathrm{T}$ cells in early pregnancy subjects and spontaneous abortion cases. Mol Hum Reprod. 2004;10:347-53. [DOI:10.1093/molehr/gah044] [PMID]

3. Wang W, Hao C, Qu Q, Wang X, Qiu L, Lin Q. The deregulation of regulatory $\mathrm{T}$ cells on interleukin-17producing $\mathrm{T}$ helper cells in patients with unexplained early recurrent miscarriage. Hum Reprod 2010;2591-6. [DOI:10.1093/humrep/deq198] [PMID]

4. Vassiliadou N, Searle R, Bulmer J. Elevated expression of activation molecules by decidual lymphocytes in women suffering spontaneous early pregnancy loss. Hum Reprod. 1999;14:1194-200.

[DOI:10.1093/humrep/14.5.1194] [PMID]

5. Lee SK, Kim JY, Hur SE, Kim CJ, Na BJ, Lee M, et al. An imbalance in interleukin-17-producing $\mathrm{T}$ and Foxp3+ regulatory $\mathrm{T}$ cells in women with idiopathic recurrent pregnancy loss Hum Reprod. 2011;2964-2971. [DOI:10.1093/humrep/der301] [PMID]

6. Walia GK, Mukhopadhyay R, Saraswathy KN, Puri M, Chahal SMS. Immuno-Molecular Etiology of Recurrent Pregnancy Loss and the Anthropological Perspective. Int J Hum Genet. 2008;8:227-35. [DOI:10.1080/09723757.2008.11886033]

7. Robertson SA, Sharkey DJ. Seminal fluid and fertility in women. Fertility and Sterility. 2016;106(3):511-9. [DOI:10.1016/j.fertnstert.2016.07.1101] [PMID]

8. Schjenken JE, Robertson SA. Seminal Fluid Signalling in the Female Reproductive Tract: Implications for Reproductive Success and Offspring Health. In: Bronson R, editor. Male Role in Pregnancy Loss and Embryo Implantation Failure. Advances in Experimental Medicine and Biology. 8682015. p. 127-58. [DOI:10.1007/978-3-31918881-2_6] [PMID]

9. Schuberth HJ, Taylor U, Zerbe H, Waberski D, Hunter R, Rath D. Immunological responses to semen in the female genital tract. Theriogenology. 2008;70(8):1174-81. [DOI:10.1016/j.theriogenology.2008.07.020] [PMID]

10. Schumacher A, Zenclussen AC. The Paternal Contribution to Fetal Tolerance. In: Bronson R, editor. Male Role in Pregnancy Loss and Embryo Implantation Failure. Advances in Experimental Medicine and Biology. 8682015. p. 211-25. [DOI:10.1007/978-3-319-18881-2_10] [PMID]

11. Martin-Villa JM, Longás J, Arnáiz-Villena A. Cyclic expression of HLA class I and II molecules on the surface of purified human spermatozoa and their control by serum inhibin B levels. Biology of Reproduction. 1999;61(6):1381-6. [DOI:10.1095/biolreprod61.6.1381] [PMID]

12. Chiang MH, Steuerwald N, Lambert H, Main EK, Steinleitner A. Detection of human leukocyte antigen class I messenger ribonucleic acid transcripts in human spermatozoa via reverse transcription-polymerase chain reaction. Fertility and sterility. 1994 Feb 1;61(2):276-80. [DOI:10.1016/S0015-0282(16)56517-0]

13. MartinVilla JM, Luque I, MartinezQuiles N, Corell A, Regueiro JR, Timon M, et al. Diploid expression of human leukocyte antigen class I and class II molecules on spermatozoa and their cyclic inverse correlation with inhibin concentration. Biology of Reproduction. 1996;55(3):620-9. [DOI:10.1095/biolreprod55.3.620] [PMID]

14. HAAS Jr GG, NAHHAS F. Failure to identify HLA ABC and Dr Antigens on human sperm. American journal of reproductive immunology and microbiology. 1986 Feb;10(2):39-46. [DOI:10.1111/j.1600-0897.1986.tb00008.x] [PMID]

15. Ohashi K, Saji F, Kato M, Wakimoto A, Tanizawa O. HLA expression on human ejaculated sperm. American Journal of Reproductive Immunology. 1990;23(1):29-32. [DOI:10.1111/j.1600-0897.1990.tb00665.x] [PMID]

16. Paradisi R, Neri S, Pession A, Magrini E, Bellavia E, Ceccardi S, et al. Human leucocyte antigen I expression in spermatozoa from infertile men. International Journal of Andrology. 2001;24(1):8-14. [DOI:10.1046/j.13652605.2001.00256.x] [PMID]

17. Paradisi R, Neri S, Pession A, Magrini E, Bellavia E, Ceccardi S, et al. Human leukocyte antigen II expression in sperm cells: Comparison between fertile and infertile men. Archives of Andrology. 2000;45(3):203-13. [DOI:10.1080/01485010050193986] [PMID]

18. Liu CD, Wang XZ, Sun XB. Assessment of sperm antigen specific $\mathrm{T}$ regulatory cells in women with recurrent miscarriage. Early Human Development. 2013;89(2):95100. [DOI:10.1016/j.earlhumdev.2012.08.003] [PMID]

\section{How to Cite This Article}

